\title{
VALORACIÓN DE LOS EFLUENTES DEL PROCESO DE DESAMARGADO DE Lupinus Mutabilis Sweet (PÓSTER)
}

\author{
$\underline{\text { Giovana Parra }}^{a^{*}}, \underline{\text { Caterine Donoso }}^{b}$, Yulisa Trávez ${ }^{b}$, Melany Llusha $^{b}$ \\ ${ }^{a}$ Universidad de Almería, España (Almería, giovana.parra@utc.edu.ec) \\ ${ }^{b}$ Facultad de Ciencias Agropecuarias y Recursos Naturales, Universidad Técnica de Cotopaxi UTC, \\ Ecuador(Latacunga, caterine.donoso7536@utc.edu.ec)
}

\begin{abstract}
Resumen
El aprovechamiento de los efluentes generados en la agroindustria es un tema de interés mundial debido al riesgo que representa su gestión inadecuada para el recurso hídrico, por esta razón el presente proyecto de investigación tiene como propósito la caracterización de los efluentes del proceso de desamargado de cuatro materiales genéticos de Lupinus mutabilis Sweet por tres métodos: tradicional, fermentación y germinación; evaluando para cada método el consumo y calidad de agua (parámetros físicoquímicos y microbiológicos) para determinar su carga residual. Además, se ha cuantificado el porcentaje de alcaloides en los efluentes con el propósito de valorar su extracción y potencial uso como biocida para control sanitario en cultivos en una investigación posterior. Los resultados evidencian que la fermentación combinada con dos lavados, es el método más eficiente para desamargar el grano, con este se consigue una remoción del 98,9\% de alcaloides, empleando $10 \%$ v/v de levadura del género Saccharomyces cerevisiae. De igual manera este método reporta un menor consumo de agua 53 litros por cada kilogramo procesado y todos los parámetros físicoquímicos y microbiológicos dentro de normativa ecuatoriana con un índice de calidad ISQA 93,90.

Palabras claves: Lupinus mutabilis Sweet, desamargado, recurso hídrico, economía agraria.
\end{abstract}

\section{Introducción y objetivos}

El cambio climático ha motivado a la industria agroalimentaria a generar prácticas de producción sustentables en cuanto a fuentes alimenticias eficientes que proporcionen el valor nutricional adecuado y a la vez su huella ecológica sea mínima. Este es el caso de algunas legumbres que constituyen fuente de proteínas para el consumo humano y cuyo impacto sobre el medio ambiente es inferior al ocasionado por el ganado [Foyer et al. (2016)]. De manera tradicional la soja ha predominado como alimento y forraje a nivel mundial, sin embargo otros cultivos de legumbres resultan prometedores por sus bondades alimenticias y de cultivos sostenibles como el Lupinus mutabilis Sweet originaria de los Andes y el Lupinus albus (altramuces) que se cultiva en España como alimento para los animales [Lucas et al. (2015)]. Aunque no son parientes filogenéticamente cercanos, los altramuces (Lupinus L. spp.), junto con las lentejas (Lens culinaris Medik.), son dos de los componentes dietéticos más valiosos desde el punto de vista nutricional que se consumen en todo el mundo. Se sabe que proporcionan una fuente equilibrada de componentes nutritivos, como aminoácidos esenciales, fibras, minerales y vitaminas. Las semillas de leguminosas tienen varios efectos beneficiosos para la salud, como antioxidantes, hipoglucemia, hipocolesterolemia, protección contra las enfermedades cardiovasculares y prevención del cáncer. [Rochfort et al. (2007); Campos-Vega et al. (2010)].

El valor alimentario de Lupinus y Lens depende del contenido relativo de fitoquímicos nutritivos y antinutritivos, mientras que esta última clase podría tener un impacto negativo en la digestibilidad de las semillas y la biodisponibilidad de los minerales y las proteínas [Farag et al. (2019)]. Por ejemplo, las semillas de Lupinus contienen cantidades variables de alcaloides de quinolizidina de sabor amargo (rango: 0-4\% de la semilla, p/p) que ofrecen a las plantas protección contra los insectos y las plagas; sin embargo, pueden dar lugar a una toxicidad anticolinérgica aguda en los seres humanos y requieren un procesamiento adicional para su eliminación, como la lixiviación [Yeheyis et al. (2011)]. El contenido fenólico de las semillas también varía dentro de las respectivas especies, subespecies o cultivares dependiendo del grado de maduración y de los estados de germinación [López et al. (2006)].

En este contexto, el propósito de la presente investigación es la valoración de los efluentes del proceso de desamargado de cuatro materiales genéticos de Lupinus mutabilis Sweet cosechados en dos índices de madurez (tierno y seco), empleando tres métodos: tradicional, fermentación y germinación, en cada método se evaluó el consumo de agua y los factores físicoquímicos y microbiológicos que determinan su calidad para la descarga residual sin representar un riesgo y como potencial uso para biocida. 


\section{Metodología}

\subsection{Métodos de desamargado del Lupinus mutabilis Sweet}

Para el aprovechamiento del lupino en la alimentación humana y animal es necesario extraer los alcaloides ya que imparten un sabor amargo y tienen acciones tóxicas en niveles de 2-4\% en las variedades amargas de Lupinus [Wink et al. (1995)].

El método tradicional de desamargado comprende tres etapas: hidratación, cocción y lavado que son posteriores a la limpieza y selección manual de la leguminosa. Se sumerge en agua durante 24 horas para su hidratación, luego se lo lleva a cocción durante 40 minutos y finalmente se realiza una secuencia de lavados, entre 10 y 12 veces, con agua clorada a $40^{\circ} \mathrm{C}$ para eliminar microorganismos, este proceso acompañado de la agitación toma un tiempo de 72 horas.

El método germinación consiste en desinfectar las semillas con hipoclorito de sodio al $1 \%$ por 30 segundos y lavarlas tres veces con agua destilada antes de escarificarlas manualmente. Se colocan en cajas Petri para iniciar su germinación bajo un fotoperiodo de $14 \mathrm{~h}$ luz y régimen de temperatura de $20 / 15^{\circ} \mathrm{C}$ en una cámara de crecimiento Lumistell, ICP-19. La germinación se evalúa a los 3 y 6 días después de la siembra. La semilla germinada alcanza una radícula mayor a $2 \mathrm{~mm}$ de longitud. Con la germinación mejora el valor nutritivo, en las leguminosas adecuadamente germinadas, los alcaloides quinolizidínicos experimentan una disminución del $27 \%$, siendo necesario un proceso de cocción y lavado para la remoción completa de estos antinutrientes. El desamargado de la leguminosa germinada se realiza en un tiempo promedio de 40 horas y reduce el contenido de alcaloides hasta el 0,004 \% [Ullco (2019)].

El método fermentación inicia con la hidratación, para esto se sumerge $1 \mathrm{~kg}$ de la leguminosa en 3 litros de agua dejándolo reposar durante 10 horas para estadío tierno y 12 horas para estadío seco. A esta solución se le añade $20 \mathrm{~g}$ de glucosa por cada litro de agua y $10 \%$ v / v de levadura del género Saccharomyces cerevisiae. Se deja reposar durante 72 horas a $30{ }^{\circ} \mathrm{C}$, se somete a cocción colocando 5 litros de agua por cada kilogramo de leguminosa, a $92{ }^{\circ} \mathrm{C}$ durante 30 minutos para estadío tierno y durante 1 hora para esadío seco. El lavado se realiza en una proporción de 5 litros de agua por cada kilogramo de lupinus, para el índice tierno el agua se cambia cada 24 horas durante 48 horas, y para el índice seco se lo realiza cada 24 horas por 72 horas con el objetivo de extraer completamente los alcaloides. El producto se almacena en refrigeración a $4{ }^{\circ} \mathrm{C}$ [Trávez et al. (2021)].

\subsection{Caracterización físicoquímica y microbiológica}

Los parámetros de calidad que se consideraron para evaluar la idoneidad de los efluentes de desamargado del Lupinus mutabilis Sweet antes de su descarga al sistema de alcantarillado son: turbidez, temperatura, conductividad eléctrica, sólidos totales, sólidos suspendidos, potencial de hidrógeno, demanda química de oxígeno, demanda biológica de oxígeno, oxígeno disuelto, unidades formadoras de colonias expresadas como coliformes totales y fecales, además del porcentaje de alcaloides y consumo de agua por cada método de desamargado. Estos parámetros fueron analizados bajo normativa técnica ecuatoriana.

\section{Resultados}

\section{1. Índices de calidad}

En el cuadro 1 se presenta el índice de calidad para cada parámetro fue determinado por comparación con el Acuerdo Ministerial 097-A relacionado a los límites de descarga al sistema de alcantarillado público. Los resultados de análisis de la caracterización del Lupinus mutabilis Sweet muestran que el agua del desamargado por el método de fermentación son estables en las concentraciones de parámetros fisicoquímicos tales como potencial de hidrógeno, temperatura, turbidez demanda química de oxígeno, demanda bioquímica de oxígeno, sólidos suspendidos, sólidos disueltos, sólidos totales, conductividad eléctrica y oxígeno disuelto, siendo estos indicadores de calidad del agua.

Cuadro 1. Parámetros e índices de calidad de agua residual en tres métodos de desamargado de Lupinus mutabilis Sweet

\begin{tabular}{|l|c|c|c|c|}
\hline $\begin{array}{l}\text { Parámetro de } \\
\text { calidad del agua }\end{array}$ & $\begin{array}{l}\text { Método } \\
\text { tradicional }\end{array}$ & $\begin{array}{l}\text { Método de } \\
\text { fermentación }\end{array}$ & $\begin{array}{l}\text { Método } \\
\text { germinación }\end{array}$ & $\begin{array}{l}\text { Índice } \\
\text { Calidad, Método } \\
\text { de fermentación } \\
\text { (ISQA) }\end{array}$ \\
\hline $\begin{array}{l}\text { Potencial } \\
\text { hidrógeno }(\mathbf{p H})\end{array}$ & 5,1 & 5,18 & 5,5 & \multirow{2}{*}{93,90} \\
\hline Temperatura $\left({ }^{\circ} \mathbf{C}\right)$ & 19,07 & 19,23 & 19 & \\
\hline
\end{tabular}




\begin{tabular}{|l|c|c|c|}
\hline Turbidez (NTU) & 179,43 & 252,60 & 23 \\
\hline DQO & 1164 & 5524,94 & 957 \\
\hline DBO5 5 & 395,76 & 370,17 & 489 \\
\hline $\begin{array}{l}\text { Sólidos Totales } \\
\text { (mg/L) }\end{array}$ & 1855,27 & 1941,04 & 2912,08 \\
\hline $\begin{array}{l}\text { Conductividad } \\
\text { eléctrica ( } \mathbf{\mu S / c m )}\end{array}$ & 1,36 & 1,81 & 1,51 \\
\hline $\begin{array}{l}\text { Oxígeno disuelto } \\
\text { (mg/L) }\end{array}$ & 10,63 & 11,75 & 13,70 \\
\hline Alcaloides (\%p/p) & 0,24 & 0,22 & 0,14 \\
\hline $\begin{array}{l}\text { Consumo de agua } \\
\text { (L/kg de chocho) }\end{array}$ & 101 & 53 & 15 \\
\hline
\end{tabular}

Fuente: Autoras, 2021

\subsection{Análisis estadístico}

Se realizaron análisis de varianza y prueba de significación de Tukey al 5\% para las fuentes que arrojaron significación estadística en los indicadores evaluados. En cuanto a la presencia de alcaloides, en el Gráfico 1 se observa la presencia de alcaloides, donde el proceso de fermentación se ubica en el rango A con $0,31 \mathrm{mg} / \mathrm{l}$ y $0,02 \mathrm{mg} / \mathrm{l}$ en el lavado inicial y lavado final respectivamente. Cabe señalar que en los procesos de hidratación no se presentó significación estadística.

Gráfico 1. Prueba de significación de Tukey al 5 \% para alcaloides en agua residual de subprocesos de tres tipos de desamargado de Lupino

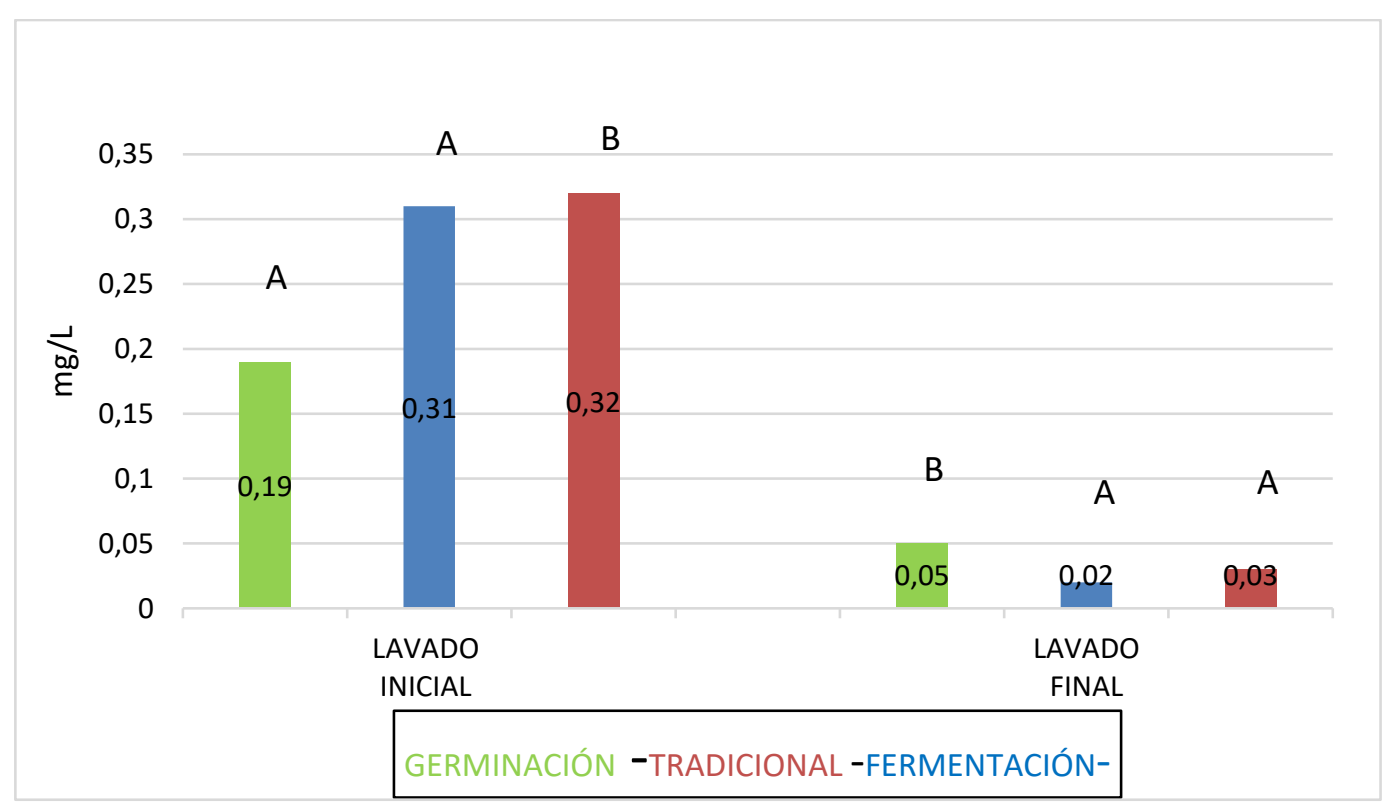

Fuente: Autoras, 2021

\subsection{Análisis económico}

Los costos totales de consumo de agua para el desamargado de un kilogramo de Lupinus mutabilis Sweet empleando los métodos: tradicional, fermentación y germinación se muestran en el Cuadro 2. Para la determinación del costo de consumo de agua en cada etapa de los tres métodos se ha considerado un costo de 0,02 USD por litro de agua empleado. Se ha considerado en el análisis el índice de madurez seco debido a que este es el que exige mayor consumo de agua en la etapa de lavado. 
Cuadro 2. Costo total por consumo de agua en subprocesos para tres métodos de desamargado

\begin{tabular}{|l|r|r|r|}
\hline \multicolumn{1}{|c|}{$\begin{array}{c}\text { Consumo de Agua } \\
\text { por etapa }\end{array}$} & $\begin{array}{c}\text { Método Tradicional } \\
\text { (USD) }\end{array}$ & $\begin{array}{c}\text { Método Fermentación } \\
\text { (USD) }\end{array}$ & $\begin{array}{c}\text { Método Germinación } \\
\text { (USD) }\end{array}$ \\
\hline Hidratación & 0,60 & 0,30 & 0,30 \\
\hline Cocción & 0,50 & 0,50 & 0,30 \\
\hline Lavado & 9,00 & 4,50 & 1,20 \\
\hline Costo Total & 10,10 & 5,30 & 1,80 \\
\hline
\end{tabular}

Fuente: Autoras, 2021

\section{Conclusiones}

Los resultados ponen de manifiesto que el mejor método de desamargado es por fermentación con una remoción del 98,9\% de alcaloides en agua residual final y un gasto de agua de 53 litros por cada kilogramo de grano procesado, lo que representa un costo total de 5,30 USD. Los parámetros de calidad del agua residual que se analizaron son: potencial hidrógeno, temperatura, turbidez, $\mathrm{DQO}, \mathrm{DBO}_{5}$, sólidos totales, conductividad eléctrica, oxígeno disuelto, alcaloides y consumo de agua. El índice de calidad ISQA obtenido 93,90 equivale a una excelente calidad de agua, estas características potencian su uso como biocida.

\section{Referencias}

Farag, M. A., Khattab, A. R., Maamoun, A. A., Kropf, M., \& Heiss, A. G. (2019). "UPLC-MS metabolome based classification of Lupinus and Lens seeds: A prospect for phyto-equivalency of its different accessions". Food Research International, 115, 379-392.

Foyer, C. H., Lam, H. M., Nguyen, H. T., Siddique, K. H., Varshney, R. K., Colmer, T. D., \& Considine, M. J. (2016). Neglecting legumes has compromised human health and sustainable food production. Nature plants, 2(8), 1-10.

Lucas, M. M., Stoddard, F. L., Annicchiarico, P., Frias, J., Martinez-Villaluenga, C., Sussmann, D., \& Pueyo, J. J. (2015). The future of lupin as a protein crop in Europe. Frontiers in plant science, 6, 705.

Rochfort, S., \& Panozzo, J. (2007). Phytochemicals for health, the role of pulses. Journal of agricultural and food chemistry, 55(20), 7981-7994.

Yeheyis, L., Kijora, C., Wink, M., \& Peters, K. J. (2011). Effect of a traditional processing method on the chemical composition of local white lupin (Lupinus albus L.) seed in North-Western Ethiopia. Zeitschrift für Naturforschung C, 66(7-8), 403-408.

López-Amorós, M. L., Hernández, T., \& Estrella, I. (2006). Effect of germination on legume phenolic compounds and their antioxidant activity. Journal of Food Composition and Analysis, 19(4), 277283.

Wink, M., Meißner, C., \& Witte, L. (1995). Patterns of quinolizidine alkaloids in 56 species of the genus Lupinus. Phytochemistry, 38(1), 139-153.

Ullco Ante, M. V. (2019). Evaluación de Estrategias Poscosecha (temperatura y desinfección) en Chocho Verde a dos Índices de Cosecha, en Campus Experimental Salache, en el periodo 2018-2019 (Bachelor's thesis, Ecuador, Latacunga: Universidad Técnica de Cotopaxi (UTC)).

Trávez, Y. \& Llusha M. (2020). Caracterización físico química del agua en el proceso de desamargado por método de fermentación del chocho (Lupinus mutabilis Sweet) en el campus experimental CEASA, provincia de Cotopaxi 2020 - 2021 (Bachelor's thesis, Ecuador, Latacunga: Universidad Técnica de Cotopaxi (UTC)).

Manotoa, G. \& Tapia M. (2020). Caracterización físico química del agua en el proceso de desamargado por el método de germinación del chocho (Lupinus mutabilis Sweet) en el campus experimental CEASA, provincia de Cotopaxi 2020 - 2021 (Bachelor's thesis, Ecuador, Latacunga: Universidad Técnica de Cotopaxi (UTC)).

Chaluisa, J. \& Jimenez M. (2020). Caracterización físico química del agua en el proceso de desamargado del chocho (Lupinus mutabilis Sweet), mediante el método tradicional en el campus experimental CEASA, provincia de Cotopaxi 2020 - 2021 (Bachelor's thesis, Ecuador, Latacunga: Universidad Técnica de Cotopaxi (UTC)). 